\title{
MITTEILUNGEN
}

\section{Das Parlament - eine Bühne für Lobbyisten? Zum Einfluss von Interessenvertretern und Experten auf politische Entscheidungen. Eine Veranstaltung der DVParl in Berlin am 22. April 2008}

Lobbyismus ist ein Thema, das in den letzten Jahren verstärkt in die öffentliche Kontroverse gerückt ist - unter anderem befördert durch Publikationen, die den Lobbyismus als „fünfte Gewalt"1 oder „stille Macht“2 oder die Bundesrepublik als „gekauften Staat“3 bezeichnen. Der Thematik stellte sich die Deutsche Vereinigung für Parlamentsfragen in einer Veranstaltung im April 2008, zu der sie einen der Herausgeber der angesprochenen Werke ebenso eingeladen hatte wie vier Lobbyisten, von denen zwei früher dem Bundestag angehört hatten, und zwei heutige Bundestagsabgeordnete. In der Diskussion zeigte sich unter anderem, dass recht verschiedene Verständnisse von Lobbyismus gepflegt werden, dass die Wichtigkeit des Parlaments unterschiedlich eingeschätzt wird und dass sich Lobbyisten in ihrer Herangehensweise und ihren Urteilen keineswegs gleichen.

Thomas Leif, Chefreporter der Chefredaktion Fernsehen des Südwestrundfunks, stellte in seinem Einführungsreferat drei Thesen vor: Erstens dominierten die übermächtige Ministerialbürokratie und die Regierung, zweitens hätte sich der Wirtschaftslobbyismus grundlegend gewandelt und professionalisiert, und drittens sei der neue Lobbyismus Ausdruck der Ökonomisierung aller Politikbereiche sowie der veränderten Managementstrukturen in der Politik. Leif grenzte den Begriff des Lobbyismus von „konventionellen lobbyistischen Tätigkeiten“ wie Anhörungen oder Fachgesprächen und auch von Nichtregierungsorganisationen ab, zählte aber den Personalaustausch zwischen Ministerien und Wirtschaft dazu. Er rief dazu auf, sich von „Parlamentsfolklore“ zu verabschieden, um endlich den Blick auf die Realitäten zu gewinnen, warnte jedoch auch, dass zunehmender Lobbyismus die Rolle des Parlaments gefährde. Zum Umgang mit Lobbyismus unterbreitete er sechs pragmatische Lösungsvorschläge: Er forderte, (1) die Empfehlungen des Bundesrechnungshofes aus einem Bericht zu dem angesprochenen Personalaustausch umzusetzen, (2) Transparenz durch einen Ehrenkodex, Jahres- und Halbjahresberichte und die Dokumentation von Gesetzgebungsprozessen (legislative Fußspuren) zu erreichen, (3) eine Abkühlungsphase bei Wechseln aus der Regierung oder dem Parlament zu Lobbyorganisationen einzuführen, (4) die empirischen Defizite in der Wissenschaft zu beseitigen, (5) eine gründlichere Arbeit der Medien sowie (6) ein stärkeres Engagement der Bürger.

Stand bei Leif eher die unrechtmäßige Beeinflussung von politischen Entscheidungen im Vordergrund, hob Cornelia Yzer, Hauptgeschäftsführerin des Verbandes Forschender Arzneimittelhersteller und Parlamentarische Staatssekretärin a.D., die Legitimität von Interes-

1 Thomas Leif/ Rudolf Speth (Hrsg.), Die fünfte Gewalt. Lobbyismus in Deutschland, Wiesbaden 2006.

2 Dies. (Hrsg.), Die stille Macht. Lobbyismus in Deutschland, Wiesbaden 2003.

3 Sascha Adamek / Kim Otto, Der gekaufte Staat. Wie Konzernvertreter in deutschen Ministerien sich ihre Gesetze selbst schreiben, Köln 2008. 
senvertretung in einer pluralistischen Gesellschaft hervor. Lobbyisten machten zudem keine Politik, selbst wenn manche dies behaupteten, sondern lieferten Abgeordneten Informationen für ihre Entscheidungen. Wie Leif forderte sie ein Höchstmaß an Transparenz und sprach sich dabei für eine feste Registrierung mit Pflichtangaben beim Bundestagspräsidenten aus. Auch ein Verhaltenskodex könne weiterhelfen, solange er prägnant und unbürokratisch formuliert sei. Sie betonte das bestehende Interesse eines Lobbyisten an Öffentlichkeit, da er Multiplikatoren für seine Anliegen suche, und dass Politiker „keine blinden Umsetzer oder Sachwalter von Interessen" seien.

Wie Yzer und nachfolgend Norbert Theihs und Jörn Thießen sah auch Wolf-Dieter Zumpfort, Direktor der TUI AG und früherer Bundestagsabgeordneter (1979 bis 1983), Lobbyismus als normalen Bestandteil von Demokratie. Er empfand Leifs Ausführungen als nicht seriös. So behandele der von ihm zitierte Sonderbericht des Bundesrechnungshofes ausschließlich den Austausch zwischen Bürokratien und Wirtschaft und befasse sich nicht mit dem täglich betriebenen Lobbyismus, zu dem Zumpfort sehr wohl auch Nichtregierungsorganisationen zählte. So wie seine Vorrednerin stellte auch er den Informationsaustausch zwischen Lobbyisten und Abgeordneten in den Vordergrund. Die Entscheidungen müssten letztere immer selbst treffen. Ähnlich argumentierte auch Norbert Theihs, Geschäftsführer der Verbindungsstelle des Verbandes der Chemischen Industrie zu Parlament und Bundesregierung. Lobbyismus sei legal und könne nur auf der Beratungs- und nicht auf der Entscheidungsebene greifen. Vernünftige Entscheidungen könnten allerdings nur getroffen werden, wenn die Akteure mit guten Informationen ausgestattet sind. Erfolg habe der Lobbyist dabei nur, wenn er die besseren Argumente auf seiner Seite hat oder seine Positionen besser begründen kann.

Karl Jurka, Inhaber einer GmbH für Politik- und Marketingberatung, vertrat andere Positionen und zeichnete auch ein anderes Bild des Lobbyismus: So sei eine Bühne, also Öffentlichkeit, nichts für Lobbyisten, und er schreibe durchaus gern Gesetze selbst. Im Gegensatz zu seinen Vorrednern, die sich als Berater beschrieben hatten, sah er sich in der Rolle, Einfluss auszuüben und Abläufe direkt beeinflussen zu können. Er definierte Lobbyisten als Übersetzer zwischen Politik und Wirtschaft, zählte auch die so genannte public diplomacy der Botschaften zum Lobbyismus und sah eine der Ursachen in der Professionalisierung des Lobbyismus auch in der gesteigerten Qualität der deutschen Beamten. Den Bundestag selbst habe er für seine Tätigkeit nur äußerst selten gebraucht. Hier zeigte sich ein fundamentaler Unterschied, der auch im Folgenden nicht geklärt werden konnte, hinsichtlich der Frage, welche Position dem Parlament im Lobbying zukommt. Ist es eher zweitrangig, wie Leif und Jurka nahelegten, oder spielt es eine nicht zu unterschätzende Rolle und ist Anlaufpunkt für Interessenvertreter, wie es die Äußerungen der anderen Lobbyisten und der zwei Parlamentarier vermuten ließen?

Die zwei derzeitigen Bundestagsabgeordneten Laurenz Meyer aus der CDU/CSU- und Jörn Thießen aus der SPD-Fraktion befürworteten jedenfalls beide Lobbyismus, berichteten aber auch von eher wenig zufrieden stellenden Zusammentreffen mit Interessenvertretern. So beschrieb Thießen Zeitverschwendung als größte Sünde des Lobbyismus und beklagte Komplexitätsreduktion und eine Unterschätzung des Bildungshungers der Parlamentarier durch die Interessenvertreter. Für ihn als Abgeordneten müsse ein Kooperationsgewinn aus dem Kontakt mit einem Lobbyisten erwachsen, und dieser ergebe sich nur, wenn letzterer auch über Einwände informiert. Lobbyismus selbst behaupte zudem oft, Politik zu machen, weil dies sein Geschäftsmodell bilde. Meyer beschrieb Lobbyismus als Rückkopplungspro- 
zess, da Abgeordnete über Lobbyisten auch über Missstände bei den Auftraggebern informieren könnten. Zudem wüssten Parlamentarier genau, was in einem Ministerium geschieht, wenn sie mit einer bestimmten Materie befasst sind; trotzdem sei es manchmal nötig, noch zusätzliche Informationen von Lobbyisten zu erhalten. So sahen auch beide, Meyer wie Thießen, Lobbyismus insgesamt als eine gute Art der Interessenvermittlung.

In seiner Replik lobte Leif die Offenheit der Diskussion, warnte aber davor, das Stimmungsbild in der Bevölkerung falsch einzuschätzen. Zudem widersprach er dem am Abend oft benutzten Begriff der Information, da das größte Problem in der Werthaltigkeit von Informationen liege. Sein Idealbild eines Abgeordneten, das - wie er einräumte - naiv sein könnte, gehe von einem in seinem Fachgebiet autonom Handelnden aus, der auf Informationen von Lobbyisten wenig angewiesen ist. Das Wichtigste sei allerdings, dass ein sauberes Lobbygeschäft ohne eine „ethische Innenausstattung“ nicht möglich sei.

In der allgemeinen Diskussion machte Ludger-Anselm Versteyl auf die „berufliche Herkunftsverfilzung" aufmerksam, in der sich Allgemein- und Individualinteressen miteinander verweben. Für ihn gehöre guter Lobbyismus außerdem in die Hearings, die seiner Ansicht nach eine Idealform der Wissens- und Informationsvermittlung darstellen. Josef Isensee sah dies skeptischer, da solche Anhörungen hohle Rituale geworden seien. Prinzipiell ist eine Parlamentslobby für ihn ein Ruhmesblatt des Parlamentarismus, wobei er zu den Lobbyisten auch Amtslobbies wie Kreis- und Gemeindetage zählte. Torsten Wolfgramm bevorzugte nichtöffentliche gegenüber öffentlichen Anhörungen, da sie meist mehr Ertrag brächten. Hier wie auch bei jedem Referentenentwurf, zu dem sie nicht nur die euphorischen Stimmen anhören sollten, fiele allein den Abgeordneten die Aufgabe und Pflicht der Abwägung zu. Wie andere zuvor trat Nils Diederich vehement dafür ein, auch Nichtregierungsorganisationen zu den Lobbyisten zu zählen; insbesondere sie seien häufig die heftigsten Lobbyisten, wenn es um Subventionen, Projektförderungen und ähnliches gehe. Eine Abkühlphase hielt auch er für sinnvoll; Wechsel aus den Ministerien in die Wirtschaft seien aber generell nicht problematisch, sondern sinnvoll. Peter Lösche war überrascht vom reformistischen Auftreten des Podiums und vom bestehenden Konsens bezüglich Transparenz. Das Problem sei nicht der Lobbyismus, sondern das über ihn bestehende Gerücht, dass Industrie, Wirtschaft und Gewerkschaften die Politik in der Hand hätten. Er vermutete allerdings, dass einige Äußerungen vielleicht nur dem Raum geschuldet seien. Daher fragte er die zwei Abgeordneten auf dem Podium direkt nach ihrer Haltung zu einem Lobbyismustransparenzgesetz, dessen Offenlegungspflichten ähnlich wie bei der Parteienfinanzierung gegenüber dem Bundestagspräsidenten bestehen könnten. Meyer und Thießen antworteten auf die Frage der Durchsetzbarkeit abwartend, da solch ein Entwurf erst geprüft werden müsste.

So lieferte der Abend insgesamt ein gemischtes Bild: Einerseits wurde größtenteils auf die Notwendigkeit und Legitimität von Lobbyismus hingewiesen, andererseits kamen mögliche „unsaubere“ Praktiken oder Missstände nur beim Hauptreferenten vor, die dieser allerdings polemisch überspitzte und thematisch so verengte, dass sich die folgenden Redner vor allem in der Rolle wiederfanden, Grundlagen der pluralistischen Demokratie zu vergegenwärtigen und zu verteidigen. Das so von den meisten in dieser Veranstaltung alles in allem positiv gezeichnete Bild des Lobbyismus in Deutschland bedarf jedenfalls weiterer glaubwürdiger Überzeugungsarbeit. 